\title{
A Versatile Cell Death Screening Assay Using Dye-Stained Cells and Multivariate Image Analysis
}

Tony J. Collins, ${ }^{1, *}$ Jarkko Ylanko, ${ }^{2, *}$ Fei Geng, ${ }^{3}$ and David W. Andrews ${ }^{2}$

${ }^{1}$ David Braley Human Stem Cell Screening Facility, Stem Cell and Cancer Research Institute, McMaster University, Hamilton, Ontario, Canada.

${ }^{2}$ Department of Biological Sciences, Sunnybrook Research Institute, Toronto, Ontario, Canada.

${ }^{3}$ School of Engineering Technology, McMaster University, Hamilton, Ontario, Canada.

*These two authors contributed equally to this work.

\begin{abstract}
A novel dye-based method for measuring cell death in imagebased screens is presented. Unlike conventional high- and medium-throughput cell death assays that measure only one form of cell death accurately, using multivariate analysis of micrographs of cells stained with the inexpensive mix, red dye nonyl acridine orange, and a nuclear stain, it was possible to quantify cell death induced by a variety of different agonists even without a positive control. Surprisingly, using a single known cytotoxic agent as a positive control for training a multivariate classifier allowed accurate quantification of cytotoxicity for mechanistically unrelated compounds enabling generation of dose-response curves. Comparison with low throughput biochemical methods suggested that cell death was accurately distinguished from cell stress induced by low concentrations of the bioactive compounds Tunicamycin and Brefeldin A. High-throughput image-based format analyses of more than 300 kinase inhibitors correctly identified 11 as cytotoxic with only 1 false positive. The simplicity and robustness of this dye-based assay makes it particularly suited to live cell screening for toxic compounds.
\end{abstract}

\section{INTRODUCTION}

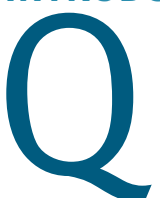

uantification of cytotoxicity is a common readout for many drug discovery endeavors. ${ }^{1}$ Programmed cell death occurs in response to a range of stresses or signals and results from the activation of one or more signaling cascades, including those characteristic of apoptosis, anoikis, necrosis, necroptosis, and autophagic cell death, and the limitations of the various current assays have been recently reviewed. ${ }^{2,3}$ Furthermore, death is generally cell autonomous and results in loss of cell adhesion complicating image-based assays of adherent cells. Detachment from the growth support or neighboring cells is not only a cell death response but also leads to cell death through anoikis; therefore, flow-based methods can overestimate cytotoxicity.

While many assays have been developed to quantify specific aspects of cell death, it has been suggested that to detect the broad spectrum of cell death cascades with high sensitivity, measurements of multiple relatively early indicators should be integrated. ${ }^{3}$ Such an approach is generally impractical for highcontent screening because of the cost and time associated with multiple often incompatible assays. Most techniques for imagebased analysis of the effects of small-molecule compounds use techniques such as immunostaining that are expensive, require extensive optimization, and are incompatible with living cells, ${ }^{4}$ or multiple dyes necessitating fixation and multiple processing steps (typically 5-10 steps in commercial kits). ${ }^{5}$

We propose an alternative image-based cytotoxicity assay for adherent cells that integrates measurement of organelle ultrastructural changes and alterations in mitochondrial function associated with programmed cell death. Unlike many cell death assays, this method uses only two dyes that can be added to cells together without a washing step, requires minimal handling or optimization, and is easily analyzed using multivariate methods available in multiple commercial

(c) Collins et al. 2015; Published by Mary Ann Liebert, Inc. This Open Access article is distributed under the terms of the Creative Commons Attribution Noncommercial License (http://creativecommons.org/licenses/by-nc/4.0/) which permits any noncommercial use, distribution, and reproduction in any medium, provided the original author(s) and the source are credited. 
and open-source software packages to enable quantification of single cells. Multivariate image analysis algorithms attempt to integrate as much of the information of each cell that can be extracted. This approach takes a broad variety of measurements (referred to as "features") from each cell to obtain a "feature-fingerprint." These are then compared to reference "feature-fingerprints," and each cell is classified to the closest matching reference dataset. Using these techniques, subcellular localization of proteins ${ }^{6,7}$ cellular subpopulations, ${ }^{8}$ and drug mechanism of action ${ }^{4,5,9}$ have been correctly classified with often greater than 95\% accuracy.

In this study, we describe a simple approach to quantify cytotoxicity in adherent cells based on multivariate analysis of cells stained with the inexpensive dye, nonyl acridine orange (NAO), and a nuclear stain (MVA-NAO). NAO is a lipophilic cationic dye with some preference for binding cardiolipin and has been shown to also be partially sensitive to the mitochondrial membrane potential. ${ }^{10}$ We compare MVANAO classification with more traditional measures of apoptosis and find that it provides improved classification in screening, quantified as improved $Z^{\prime}$ factor $\left(Z^{\prime}\right)$, a standard screening assay metric. Moreover, this dye combination can be used to quantify $\mathrm{EC}_{50}$ values when used in a dose-response format. With an average cost that ranges from $\$ 0.1-10$ per plate (depending on the nuclear stain), compared to commercial kits that average $\$ 50$ per plate, this method is particularly well suited for applications involving large numbers of samples, such as high-content screening.

\section{MATERIALS AND METHODS}

Cell Culture and Reagents

Human breast cancer cells MCF-7 were maintained in the $\alpha$-minimal essential medium ( $\alpha$-MEM; Life Technologies, Carlsbad, CA) supplemented with 10\% fetal bovine serum (HyClone, Logan, UT). Cells were seeded, treated, and stained under Biosafety level 2 conditions using a custom Sample Preparation WorkCell platform (Thermo Fisher CRS, Burlington, ON, Canada) that contains a CRS VAL 3-axis robot (Thermo Fisher) for plate handling, Combi Multidrop dispensers (Thermo Fisher), ELx plate washers (Biotek, Winooski, VT), and a STARlet 96-tip robot (Hamilton, Reno, NV) for precise liquid dispensing. Cells were seeded at a cell density of 5,000 cells per well to a 384-well plate (Cell Carrier; PerkinElmer, Waltham, MA) and grown for $24 \mathrm{~h}$ before drug treatment, or transfected with a plasmid encoding the caspase sensor (a kind donation from Dr. R. Truant, McMaster University) using NanoFect (QIAGEN, Venlo, Limburg, Netherlands) reagent according to the manufacturer's standard protocol, $24 \mathrm{~h}$ before drug treatment.
Drug Treatments

Drugs were dissolved in (DMS0; Bioshop, Burlington, ON, Canada) and diluted to the final assay concentrations in $\alpha$-MEM. Tumor necrosis factor-alpha (TNF $\alpha$ ), cycloheximide, thapsigargin (TG), brefeldin A (BFA), dithiothreitol (DTT), actinomycin D, staurosporine, tamoxifen, and tunicamycin, were purchased from Sigma-Aldrich (St. Louis, M0). The panel of 320 kinase inhibitors was obtained from the Ontario Institute for Cancer Research.

\section{Cell Staining}

Cells were incubated under culture conditions with fluorescent dyes for $30 \mathrm{~min}$ before image acquisition, using either $100 \mathrm{nM}$ NAO (Sigma-Aldrich) or $10 \mathrm{nM}$ tetramethylrhodamine, ethyl ester (TMRE; Life Technologies) in $\alpha$-MEM, or $1 \mu \mathrm{M}$ propidium iodide (PI; Sigma-Aldrich) and 1:100 Annexin V Alexa Fluor 488 (Life Technologies) in 0.01 M HEPES $\mathrm{pH} 7.4,0.14 \mathrm{M} \mathrm{NaCl}$, and $2.5 \mathrm{mM} \mathrm{CaCl}_{2}$. For the experiments reported here we used $5 \mu \mathrm{M}$ DRAQ 5 (Biostatus, Leicestershire, UK) as a nuclear dye for cell identification and segmentation; however, we have successfully used other nuclear dyes, including Hoescht and DAPI for this purpose.

\section{Biochemical Assays}

For analysis of poly ADP-ribose polymerase (PARP) cleavage, light chain 3 (LC3), and glucose-regulated protein (Grp) 78 and Grp94 levels, proteins were separated on 8\% denaturing sodium dodecyl sulfate-polyacrylamide gel electrophoresis (SDS-PAGE) gels and transferred to polyvinyl difluoride membrane, incubated with a 1:1,000 dilution of PARP C-2-10 monoclonal antibody or KDEL antibody to detect Grp78 and Grp94 from Enzo Life Sciences (Farmingdale, NY), LC3 antibody from Cell Signaling Technologies (Danvers, MA), and the antibody to actin used for normalization was from Santa Cruz Biotechnology (Dallas, TX). These antibodies were detected with a 1:10,000 dilution of the corresponding horseradish peroxidase-conjugated secondary antibody from Jackson ImmunoResearch Laboratories (West Grove, PA), and Enhanced Chemiluminescence reagents (EMD Millipore, Billerica, MA) and band intensities were recorded using a CCD camera-based imager (MicroChemi 4.2; FroggaBio, Toronto, ON, Canada). Where required, band intensities were corrected for unequal loading by reprobing the blots for actin.

\section{Microscopy}

All cells were imaged at $37^{\circ} \mathrm{C}$ and $5 \% \mathrm{CO}_{2}$ using an Opera QEHS high-content screening system (PerkinElmer) with environmental control for the imaging chamber and a tunable Ti:Sapphire pulsed laser and frequency doubler from Coherent 
(Santa Clara, CA). Images were acquired using $40 \times 0.9 \mathrm{NA}$ and $20 \times 0.7 \mathrm{NA}$ water immersion objectives. Data shown were recorded with the $40 \times$ lens, however, similar results were obtained with either lens. Images of NAO and Annexin V Alexa Fluor 488 staining were acquired with $488 \mathrm{~nm}$ excitation and 520/35 emission filter; PI and TMRE with $561 \mathrm{~nm}$ excitation and 600/40 emission; DRAQ5 with $633 \mathrm{~nm}$ excitation and 690/ $50 \mathrm{~nm}$ emission. Because the fluorescence intensity of NAO is as high as most nuclear stains, it is possible to image 384-well plates rapidly. Generally, for imaging multiple fields of view per well (here 3-8), the time depends primarily on the instrument used and typically ranges from 15 to $30 \mathrm{~min}$. Fluorescence images of monomeric Cerulean (mCer) in the caspase sensor were collected with $430 \mathrm{~nm}$ nanosecond pulsed excitation and 475/50 emission, using a fast gated CCD camera. For ratiometric Förster resonance energy transfer (FRET), mCer was excited as above and fluorescence was recorded from the Venus fluorescence protein using the 520/35 emission filter. well averages calculated for each feature. Support vector machine (SVM) classifiers were trained (MATLAB function svmtrain()) using well-level data and then used to classify celllevel data (svmclassify()). The fraction of individual cells per well per classified group was calculated. Various measures of cell death were compared by Z-factor analysis, using the mean and standard deviation of eight untreated negative control and four TNF $\alpha$-treated positive control replicate wells per assay.

\section{RESULTS}

MVA-NAO of Images of Dye-Stained Cells Is More Sensitive and More Robust than Standard Fluorescence Imaging Methods for Measuring Cell Death

Several conventional image-based assays used to quantify cell death are illustrated for MCF-7 breast cancer cells $24 \mathrm{~h}$ post-treatment with TNF $\alpha$ and cycloheximide (referred to here as TNF $\alpha$ treatment), a standard method for inducing extrinsic apoptosis (Fig. 1). Annexin V binding measures the externalization of phosphatidylserine that occurs during apoptosis
Image and Data Analysis

Images collected on the Opera were analyzed using Acapella 2.5 (PerkinElmer). Assay-specific features of fluorescence intensity and texture features, including Haralick texture ${ }^{10}$ features and threshold adjacency statistics, ${ }^{6}$ were calculated using custom algorithms (scripts available from www.andrewslab.ca). Similar methods are available in commercial software packages, including Acapella, Harmony, and Columbus, and in open source programs such as CellProfiler and OME. We have found that multiple software packages perform equally well with the images analyzed here. Cell masks were identified from DRAQ5 images with Acapella routines that perform similar to those in CellProfiler. A full list of the 235 features used is available from the authors. Values for each cell were generated and exported to MATLAB for further analysis.

In MATLAB, the cell-level data were transformed to $z$-scores and

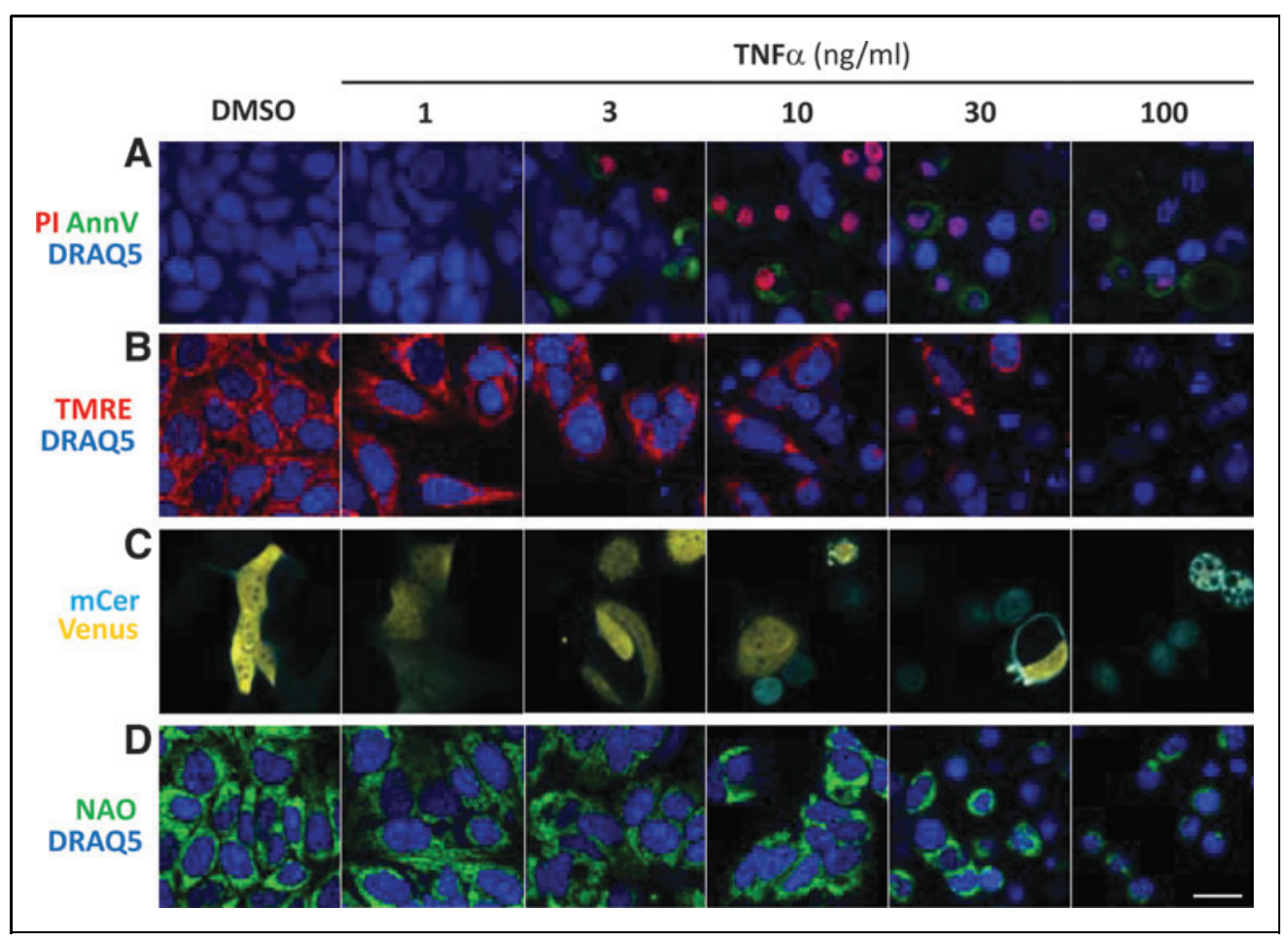

Fig. 1. Image-based cell death assays. Example images of cells $24 \mathrm{~h}$ after TNF $\alpha$ treatment by the fluorescence assays under evaluation. For each assay, the relevant channels were merged, pseudocolored, and adjusted for brightness and contrast. (A) Cells stained with propidium iodide (PI, red), Annexin V Alexa Fluor 488 (AnnV, green), and DRAQ5 (blue). (B) Cells stained with tetramethylrhodamine, ethyl ester (TMRE; red), and DRAQ5 (blue). (C) Intensity weighted ratio images of cells expressing the caspase sensor showing monomeric Cerulean (mCer; cyan) and stimulated emission of Venus (Venus, yellow). (D) Cells stained with nonyl acridine orange (NAO; green) and $\mathrm{DRAQ}_{5}$ (blue). Scale bar $=20 \mu \mathrm{m}$. 
when cells no longer retain the plasma membrane lipid asymmetry. However, as seen from the images in Figure 1, Annexin $\mathrm{V}$ binding was useful for a limited number of TNF $\alpha$ treatments, because at higher doses staining decreases as cells fragment and die. PI staining measures the loss in integrity of the plasma membrane that occurs early in necrosis, but late during apoptosis, but also has a narrow detection range (Fig. 1A). TMRE, a cell-permeant, cationic, red-orange fluorescent dye sequestered by active mitochondria and used to quantify mitochondrial membrane potential, has an intermediate detection range (Fig. 1B).

Caspase activation, a hallmark of apoptosis, was measured using a Cerulean and Venus fluorescence protein FRET-based caspase reporter (Fig. 1C). Cleavage of the sensor at the caspase 3/7 site DEVD separates the donor (mCer) from the acceptor yellow fluorescence protein (Venus) abolishing FRET and decreasing the fluorescence of the acceptor. This assay reliably detects apoptosis, but no other forms of cell death, and requires the cells be transfected to express the sensor. Nuclear condensation and cell counts were derived from DRAQ5 staining in cells (Figs. 1A, B, D, and 2B).
In contrast to these conventional assays, NAO stains multiple subcellular membrane structures, emphasizing the mitochondria due to their high membrane potential and cardiolipin content and reports on cell death indirectly (Fig. $1 D$ ). Under these conditions, changes in cell ultrastructure characteristic of cell death due to TNF $\alpha$ are visually obvious, and the assay has a large detection range and requires minimal optimization.

For the conventional image-based assays, response to drug treatment was detected on a cell by cell basis by segmenting the micrographs using the DRAQ5 image and applying an intensity threshold established from the untreated samples to the other channel. As little as $10 \mathrm{ng} / \mathrm{mL}$ TNF $\alpha$ treatment was detected by all of these assays (Fig. 2). Annexin V staining was the most sensitive and allowed detection $(P<0.005)$ at $0.3 \mathrm{ng} / \mathrm{mL}$, very similar to biochemical analyses that detected caspasedependent cleavage of PARP to the characteristic cleaved form ( $\triangle$ PARP), in response to treatment with $1.0 \mathrm{ng} / \mathrm{mL} \mathrm{TNF} \alpha$ (Fig. 2F, G). The biochemical assays provide a wealth of information that is unfortunately convolved, including increased total PARP in response to DNA damage or stress,

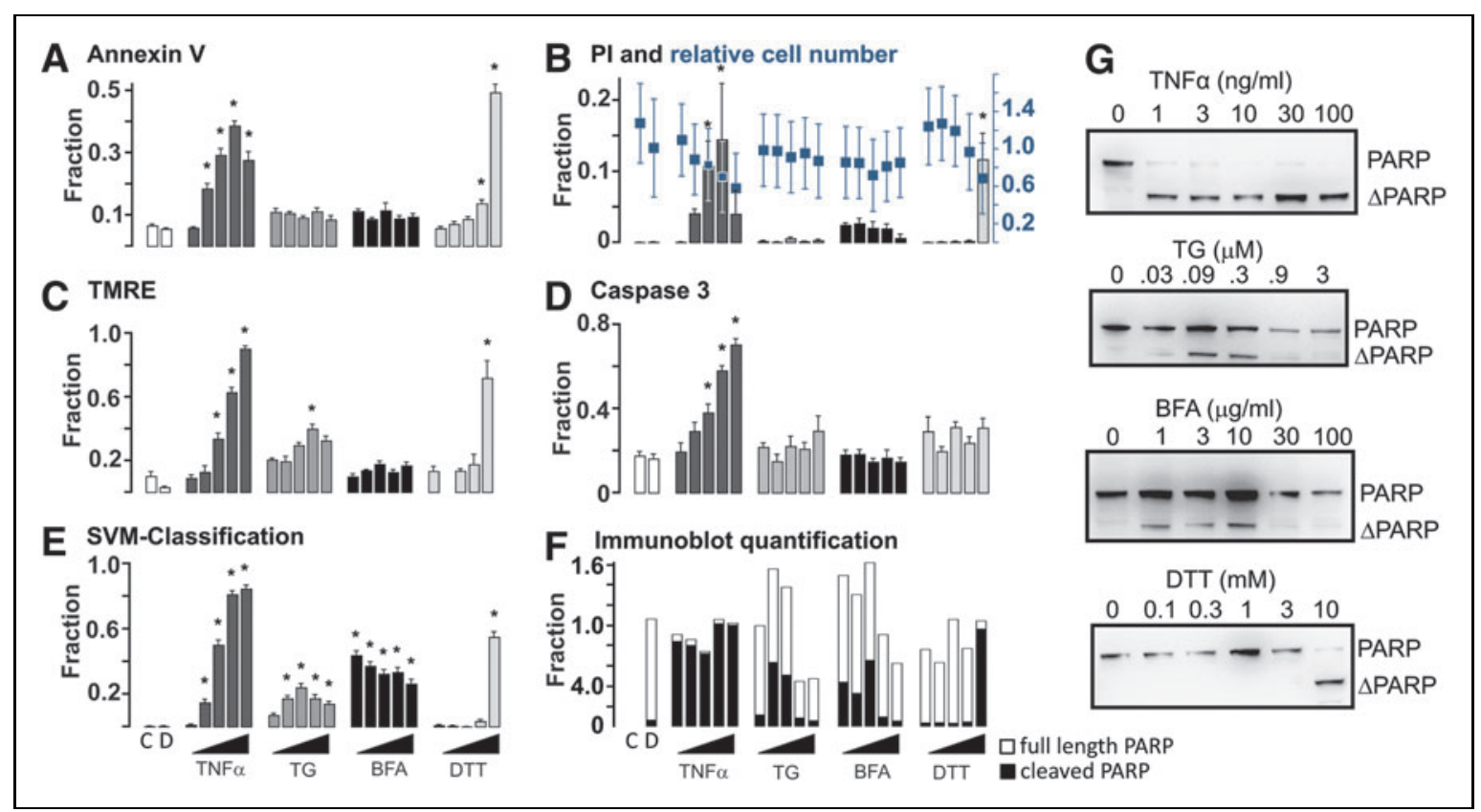

Fig. 2. Cell death measurements using conventional image-based assays are not as sensitive as MVA-NAO and immunoblotting. (A-F) Histograms illustrating the response of cells to the negative control (C) with no addition, dimethylsulfoxide (DMSO), or TNF $\alpha$ (1, 3, 10, 30, $100 \mathrm{ng} / \mathrm{mL}$ ), thapsigargin (TG; 30, 90, 300, $900 \mathrm{nM}, 3 \mu \mathrm{M}$ ), brefeldin A (BFA; 1, 3, 10, 30, $100 \mu \mathrm{g} / \mathrm{mL}$ ), and dithiothreitol (DTT; .1, .3, 1, 3, $10 \mathrm{mM}$ ) measured using the indicated assay. The fraction of cells scored as affected using an arbitrary threshold for conventional imagebased methods of quantifying apoptosis, including (A) Annexin V, (B) PI and cell number (blue), (C) TMRE, and (D) caspase activity compared to (E) MVA-NAO for a range of proapoptotic treatments. Bars indicated with * were significantly different than the negative controls ( $t$-test, $P<0.05, n=4)$. (F-G) Immunoblots for poly-ADP ribose polymerase (PARP) indicate apoptosis as cleavage of PARP to $\triangle$ PARP (migration positions indicated to the right of the blots) or necrosis as degradation of PARP (loss of both bands), (F) quantified after correction for actin levels measured from the same blots. 
cleaved PARP due to apoptosis, and degraded PARP due to necrosis. ${ }^{11}$ As expected, TNF $\alpha$ treatment induced only apoptosis while most of the other drugs elicited stress, necrosis, and apoptosis. As a result, the correlation of the blotting results and the high-content methods requires close examination of the quantified data.

The fraction of Annexin V-positive cells peaked at 40\% for $\mathrm{TNF} \alpha$ treatment and decreased at higher doses due to loss of staining of the cells (Fig. 1). Other assays suggest this is an underestimate of cells undergoing cell death as caspase activation, and TMRE staining identified 70\%-90\% of cells as having responded to TNF $\alpha$ treatment (Fig. 2C, D). Interestingly, the highest concentration of TNF $\alpha$, shown by other measures to produce the highest level of cell death, results in a low measure of death as quantified by PI (Fig. 2B). As shown in Figure 1, at the latter stages of cell death there is sufficient DNA degradation to reduce PI staining leading to false negatives. Furthermore, cell count based on DRAQ5 staining was highly variable and at our assay time point was for most treatments relatively unchanged, thus limiting its usefulness to detect cell death (Fig. 2B).

Conventional image-based assays were not sensitive for detecting changes in cells induced by TG, an endoplasmic reticulum calcium pump inhibitor, BFA, a toxin that blocks forward vesicular transport between the endoplasmic reticulum and Golgi, or DTT, a reducing agent that causes protein misfolding in the endoplasmic reticulum by preventing proper disulfide bond formation. Concentrations of $0.09 \mu \mathrm{M}$ TG and to a limited extent $1-10 \mu \mathrm{g} / \mathrm{mL}$ BFA were found to induce PARP cleavage $24 \mathrm{~h}$ post-treatment in immunoblot-based assays. However, cellular responses, as assessed by PARP immunoblots, are complicated for TG and BFA as the drug induced damage results in increased PARP levels in cells, while apoptosis caused PARP to be cleaved at a site specific for caspases 3 and 7, and necrosis results in degradation of both PARP bands (Fig. 2F, G) as seen previously for other drug treatments. ${ }^{11}$

After $0.9 \mu \mathrm{M}$ treatment with $\mathrm{TG}$, a small but statistically significant response was detected using TMRE. A response to BFA treatment was not detected for any concentration using any of the conventional image-based readouts of cell death (Fig. 2A-D). These results demonstrate that conventional image-based assays fail due to the limited range of drug treatments they respond to and inaccurate scoring of cells undergoing nonapoptotic responses to drugs such as TG and BFA.

Only the highest concentration of DTT $(10 \mathrm{mM})$ showed significant change in PI positivity, TMRE negativity, and Annexin V staining. The latter was significantly different $(P<0.01)$ between control and $3 \mathrm{mM}$ DTT. Consistent with this result and expectation based on the known propensity of DTT to cause cell stress that only leads to the unfolded protein response triggering apoptosis in extreme conditions, $10 \mathrm{mM}$ DTT was also required to induce apoptosis detected biochemically (Fig. 2G). Caspase 3 positivity reliably detected only TNF $\alpha$ treatment (Fig. 2D) and death due to the highest concentration of DTT. This may be because activation of caspase 3 is a response to apoptosis and the PARP western blots reveal minimal PARP cleavage in response to TG or BFA. Instead, PARP degradation characteristic of necrosis ${ }^{2}$ is seen at the higher concentrations of these drugs (Fig. 2F, G).

To improve the detection of cytotoxicity due to all of these agents, we used MVA-NAO. Images were acquired (Table 1), and the DRAQ5 images were used to identify the cell nuclei and cytoplasmic masks. These masks were then used to quantify 235 features for each cell from the NAO and DRAQ5 images. These features included texture, intensity, shape, and size descriptors for the cytoplasm and nuclei.

A simple threshold cannot be used with multiparametric data; therefore, to process the features from NAO- and DRAQ5-stained cells, we used classification with a SVM, a standard multivariate approach (Fig. 2E). An SVM was generated that maximally classified random subsets of the control cells as either negative (untreated) or positive (treated), based on images of the cells either untreated or exposed to $100 \mathrm{ng} /$ $\mathrm{mL} \mathrm{TNF} \alpha$, respectively. Traditionally, an SVM is calculated based on a positive and negative control for each treatment. However, to mimic screening data, the images from TNF $\alpha$ treated and vehicle-treated wells were used as high and low controls to train a classifier that was then applied to all of the drug treatments. The fraction of cells classified as positive (number of positive cells/well divided by the total number of cells/well) was then expressed for each well as a measure of cytotoxicity.

MVA-NAO showed significant differences in the fraction of cells classified positively between control and $3 \mathrm{ng} / \mathrm{mL}$ TNF $\alpha$ treatment, the lowest TNF $\alpha$ concentration detected by conventional image-based approaches (compare Fig. 2A-D with $E)$. SVM classification resulted in a similar high fraction response for TNF $\alpha$ treatment as seen with TMRE and caspase activity $(\sim 0.8)$. However, unlike the conventional methods, multivariate analysis detected responses to all concentrations of BFA and to all but the lowest concentration of TG. Biochemical assays, in which caspase mediated cleavage and degradation of PARP, were used to assess apoptosis and necrotic cell death, respectively, and to detect only population averages not individual cells. Nevertheless, inspection of the immunoblots used to assess PARP cleavage and degradation suggests that the biochemical data are qualitatively most 
COLLINS ET AL.

\begin{tabular}{|c|c|c|c|}
\hline Step & Parameter & Value & Description \\
\hline 1 & Plate cells & $40 \mu \mathrm{L}$ & 5,000 cells/well \\
\hline 2 & Incubation time & $18-24 \mathrm{~h}$ & $37^{\circ} \mathrm{C}, 5 \% \mathrm{CO}_{2}$ \\
\hline 3 & Controls & $10 \mu \mathrm{L}$ & Diluted in HEPES, added at final $1 \times$ concentration \\
\hline 5 & Incubation time & $24 \mathrm{~h}$ & $37^{\circ} \mathrm{C}, 5 \% \mathrm{CO}_{2}$ \\
\hline 6 & Dye addition & $50 \mu \mathrm{L}$ & Diluted in media, added at final concentrations of $100 \mathrm{nM}$ NAO, 5 uM DRAOS \\
\hline 7 & Incubation time & $1 \mathrm{~h}$ & $37^{\circ} \mathrm{C}, 5 \% \mathrm{CO}_{2}$ \\
\hline 8 & Assay readout & $\begin{array}{l}\text { Excitations at } 488 \text { and } 635 \mathrm{~nm} \text {, } \\
\text { emissions at } 520 \text { and } 690 \mathrm{~nm}\end{array}$ & Opera QEHS \\
\hline 10 & Feature scaling & $z$-Score & $\begin{array}{l}\text { For each descriptor, feature mean scaled to } 0 \text {, and standard deviation set as whole number } \\
\text { increments from mean }\end{array}$ \\
\hline 11 & \multicolumn{3}{|l|}{ SVM classification } \\
\hline (a) & Classifier training & $\begin{array}{l}n \text { Features on } m \text { high and low } \\
\text { control wells }\end{array}$ & $\begin{array}{l}\text { Well-level averages for all features in DMSO (low control) and TNF } \alpha+C H x \text { (high control) wells; } \\
m=32\end{array}$ \\
\hline (b) & Cell-level classification & $n$ Features of $k$ query cells & $k>400$ per query well \\
\hline 12 & \multicolumn{3}{|l|}{ Euclidean distance analysis } \\
\hline
\end{tabular}

\section{Step Notes}

1. Cells dispensed at all wells of a black wall clear-bottom cell culture plate.

3. Added to columns $1,2,23$, and 24 .

4. Added to columns 3-22.

8. Imaging performed at constant $37^{\circ} \mathrm{C}, 5 \% \mathrm{CO}_{2}$.

9. A completed list of measured features is available from the authors on request.

10. Performed if positive and negative experimental controls are present.

12. Performed if only negative experimental control is present.

DMSO, dimethylsulfoxide; NAO, nonyl acridine orange; SVM, support vector machine; TNF $\alpha$, tumor necrosis factor-alpha.

similar to the results obtained using multivariate analysis of images of cells stained with NAO and DRAQ5 (Fig. 2E, F), consistent with these techniques being superior to other image-based techniques. For both TG and BFA, MVA-NAO was less sensitive at the two highest drug concentrations where the biochemical assays suggested there was significant cell death due to necrosis (degradation of both PARP bands). In contrast, the PARP immunoblots for cells treated with TNF $\alpha$ show evidence only of apoptosis (cleavage of full-length
PARP to $\triangle \mathrm{PARP}$ ). Since images of cells treated with TNF $\alpha$ were used as the training group, the absence of necrosis in the positive control may explain why the classifier was less sensitive for images of cells undergoing necrosis (Fig. 2E compared to $F$ ).

To compare the different methods quantitatively, $Z^{\prime}$-values were calculated for a range of conventional measures of cell death, including those shown in Figure $2 A-D$ and MVA-NA0. The highest performing conventional assay was TMRE with a 


\begin{tabular}{l|c} 
Table 2. Z' Calculated from Untreated and & $100 \mathrm{ng} / \mathrm{mL}$ \\
TNF $\alpha$ Treatments & $\mathbf{Z}^{\prime}$ \\
Apoptosis & 0.91 \\
\hline NAO-SVM & 0.57 \\
\hline TMRE positive & 0.34 \\
\hline Caspase-3 activity & 0.01 \\
\hline Annexin V positive & -0.3 \\
\hline Cell count & -1.38 \\
\hline NAO intensity & -4.0 \\
\hline PI positive & -27 \\
\hline Nuclear area & \\
\hline
\end{tabular}

$\mathrm{PI}$, propidium iodide; TMRE, tetramethylrhodamine ethyl ester.

$\mathrm{Z}^{\prime}$ of 0.57. Other measures such as cell count, nuclear condensation (i.e., nuclear area), and loss of membrane integrity (PI positivity) were poor screening assays with $\mathrm{Z}^{\prime}$ of less than zero in this assay format (Table 2). To derive a $Z^{\prime}$ value for MVA-NAO, we used the readily interpretable "percentage of cells per well classified as positive." The resulting $Z^{\prime}$ value of 0.91 outperformed all of the conventional image-based cell death assays (Table 2).

\section{Derivation of Dose-Response Curves Using MVA-NAO}

Secondary assays in screening campaigns often aim to derive the $\mathrm{EC}_{50}$ concentration of active compounds based on their dose-dependent effects on cells. To further test the utility of MVA-NAO for high-content screening, cells were treated with a range of concentrations of compounds chosen to target a variety of cell death or stress pathways. As above, the extrinsic apoptosis pathway was targeted by TNF $\alpha$ treatment. In addition, drugs were added to cause endoplasmic reticulum stress (tunicamycin); intrinsic apoptosis (staurosporine), ${ }^{12}$ autophagy (low-dose tamoxifen), and mitotic catastrophe (actinomycin D) ${ }^{13}$ (Fig. 3A) and the percentage of cells classified positive for each treatment were quantified. Unlike the experiments described above in which we used images of only TNF $\alpha$-treated cells as the positive controls, here we used the highest concentration of each treatment as its respective positive training set and derived $\mathrm{EC}_{50}$ values for the response (Fig. 3B). Using this approach, it was possible to generate dose-response curves for each of the cell treatments.

The dose-response curves for the drugs that induce apoptosis (TNF $\alpha$, actinomycin D and staurosporine) exhibit the expected S-shape curves with $\mathrm{EC}_{50} \mathrm{~S}$ of $5 \mathrm{ng} / \mathrm{mL}, 60 \mathrm{nM}$, and $125 \mu \mathrm{M}$, respectively, and these drugs show similar dosedependent apoptosis as assessed by the cleavage of PARP (Fig. 3C). Moreover, both actinomycin and staurosporine exhibit a marked transition in PARP cleavage at a drug concentration near the $\mathrm{EC}_{50}$ determined by NAO-MVA.

As expected, the PARP cleavage does not correlate nearly as well with the dose-response curves obtained by MVANAO for tamoxifen and tunicamycin, drugs that cause autophagy and endoplasmic reticulum stress, respectively. Biochemical assessment of autophagy for tamoxifen-treated cells by immunoblotting for activated LC3 permits detection of autophagy at $5 \mu \mathrm{M}$ and shows a sharp increase between 9.4 and $18 \mu \mathrm{M}$. Cleavage of PARP indicative of apoptosis is not detected for tamoxifen-treated cells, but degradation is pronounced by $5 \mu \mathrm{M}$. MVA-NAO was less sensitive and detects a sharp transition in phenotype at around $60-70 \mu \mathrm{M}$ tamoxifen. For tunicamycin, biochemical detection of endoplasmic reticulum stress as increased levels of Grp78 and Grp94 is apparent at $0.8 \mu \mathrm{M}$. In contrast, MVA-NAO does not detect phenotypic changes in tunicamycin-treated cells until around $10 \mu \mathrm{M}$ with an $\mathrm{EC}_{50}$ that can only be estimated in the absence of saturation of almost $80 \mu \mathrm{M}$. These results for tamoxifen and tunicamycin are consistent with MVA-NAO trained on the highest dose of the drugs detecting common features of dying cells and, therefore, being less sensitive at detecting changes due to autophagy or endoplasmic reticulum stress.

While MVA-NAO enabled comparing cell death induced by different concentrations of a single treatment, in most screens the effect of unknown compounds is compared to a single positive control. To mimic this situation, images of cells treated with the highest concentration of TNF $\alpha$ and stained with NAO and DRAQ5 were used to train an SVM, as above, which was then applied to all of the other sets of images. In this situation, the different doses of the various compounds act as surrogates for compounds with different activities. The resulting dose-response curves were surprisingly similar to those generated using a separate SVM for each drug treatment (Fig. 3D), suggesting that MVA-NAO can be used in a screening format to examine a variety of drugs suffering only a small loss in sensitivity compared to an optimized assay.

MVA-NAO Identification of Cytotoxic Kinase Inhibitors

The data above strongly suggest that MVA-NAO can be used to identify toxic compounds in libraries of small molecules. To test this directly, an annotated small-molecule kinase inhibitor library was assayed at $1 \mu \mathrm{M}$ with TNF $\alpha$ (at 


\section{COLLINS ET AL.}

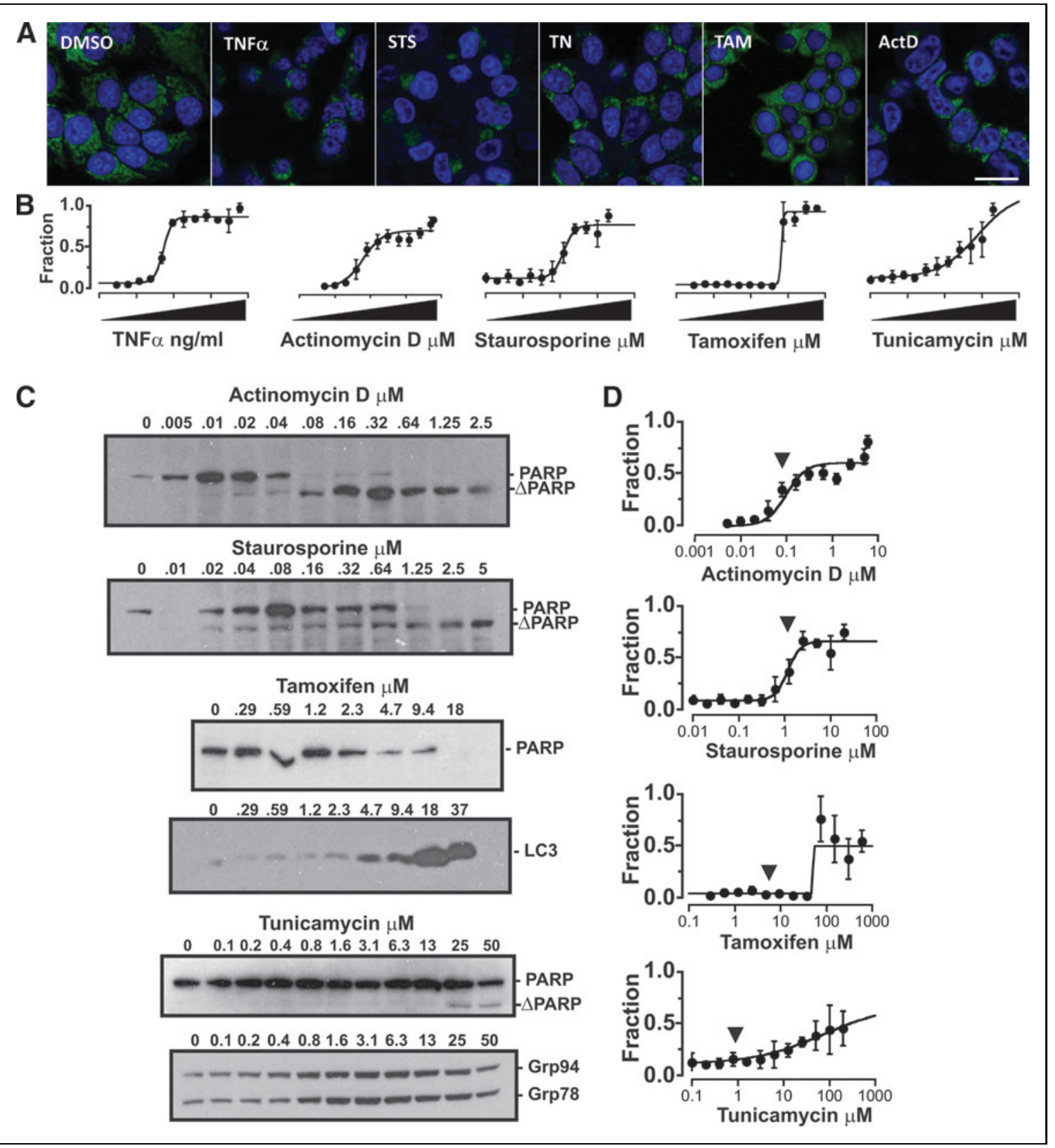

Fig. 3. Cell death dose-response curves generated for cytotoxic compounds by MVA-NAO are comparable to those obtained by immunoblotting. (A) Example images of cells $24 \mathrm{~h}$ after drug treatment. Merged, pseudocolored images of NAO (green) and DRAQ5 (blue) were adjusted for brightness and contrast. Panels show cells with no treatment (DMSO) or with $150 \mathrm{ng} / \mathrm{mL}$ TNF $\alpha, 5 \mu \mathrm{M} \mathrm{STS}, 50 \mu \mathrm{M}$ TN, $150 \mu \mathrm{M}$ TAM, and $2.5 \mu M$ ActD. Scale bar $=20 \mu \mathrm{m}$. (B) Dose-response curves generated by MVA-NAO for TNF $\alpha(0.2-500 \mathrm{ng} / \mathrm{mL})$, the cytotoxic drugs actinomycin D and staurosporine, and agents that cause autophagy (tamoxifen) or endoplasmic reticulum stress (tunicamycin). Increasing dose is indicated by the triangle, range is indicated in (D). Images of cells treated with DMSO and the highest dose of each drug were used to generate a classifier for that drug. Each point represents the average and standard deviation in fraction of cells/well classified as affected by the drug of eight replicate wells. (C) Detection of cell death, autophagy, and endoplasmic reticulum stress by immunoblotting cell lysates for PARP, light chain 3 (LC3), glucose regulated protein (Grp) 94, and Grp78 (bands are indicated to the right of the blots). Cleavage of PARP to $\triangle$ PARP indicates apoptosis, while degradation of PARP and $\triangle$ PARP indicates necrosis. Detection of the activated (cleaved and lipidated) form of LC3 indicates autophagy. Increased amounts of the abundant KDEL containing proteins Grp94 and Grp78 indicate endoplasmic reticulum stress. (D) Dose-response curves generated by MVA-NAO for the indicated drugs. Images randomly selected from the DMSO control and the highest dose TNF $\alpha$ were used to generate a classifier that was applied to images obtained for cells treated with the other drugs. Each point represents the average and standard deviation in fraction of cells/well classified as affected by the drug of eight wells. The downward arrow indicates the dose at which a change in phenotype was detected by immunoblotting. 
DYE-BASED ASSAY FOR CELL DEATH

$29 \mathrm{ng} / \mathrm{mL}$ based on Fig. 3A) and 0.1\% DMSO as high and low controls, respectively. At $1 \mu \mathrm{M}$, most of the kinase inhibitors in the library are not expected to be toxic. Afterward, drug treatment cells were stained with NA0 and DRAQ5 as above. To validate the assay for high-throughput-type experiments, where image acquisition is often the rate-limiting step, we reduced the magnification used to $20 \times$ and the numbers of fields acquired to 3 . At this magnification and resolution, the $Z^{\prime}$ was still high $\left(Z^{\prime}=0.62\right)$ and throughput was much increased (a 384-well plate could be imaged in less than $30 \mathrm{~min}$ ).

A heat map for the results illustrates the fraction of cells in each well scored as similar to the positive control (Fig. 4). Based on compound annotation for other cell lines (Table 3), we defined as toxic compounds in which more than 50\% of the cells scored similar to TNF $\alpha$. To confirm that this was a sufficiently stringent arbitrary criterion to identify toxic compounds, we treated cells with $1 \mu \mathrm{M}$ of the 11 kinase inhibitors that scored positive as above and then assessed 6 days after treatment whether cells exposed to these compounds were still viable. With the exception of Dasatinib, all of the cells treated with the compounds designated as toxic by MVA-NAO eventually died (data not shown). This result confirms that MVA-NAO can be used to identify toxic compounds in a screening-type assay.

\section{DISCUSSION}

A cell is considered dead in vitro when it loses plasma membrane integrity ${ }^{2,3}$; however, a cell can be committed to dying long before this point is reached. Initiation of cell death processes is associated with a wide range of measurable changes within the cell. ${ }^{2}$ Biochemical assays based on

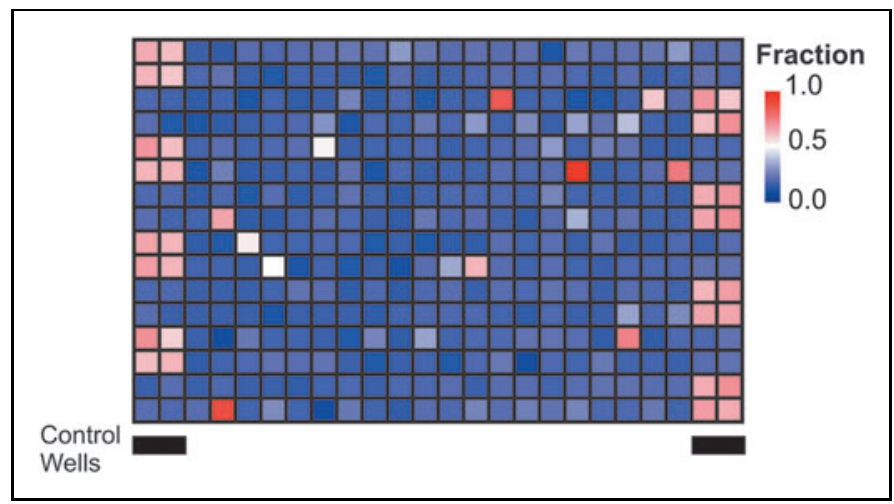

Fig. 4. Cytotoxic kinase inhibitors identified by MVA-NAO screening. MVA-NAO heat map where the color indicates the fraction of cells per well classified as affected (similar to TNF $\alpha$ treated), as indicated to the right of the panel. Columns of control wells containing cells treated with either TNF $\alpha$ or DMSO (pink or blue, respectively) are indicated below the panel.

\begin{tabular}{|c|c|c|c|c|}
\hline Compound & Targets & Fraction & Relevance & Reference \\
\hline $\begin{array}{l}\text { Alvocidib/ } \\
\text { Flavopiridol }\end{array}$ & CDK & 0.98 & $\begin{array}{l}\text { Chronic lymphocytic } \\
\text { leukemia }\end{array}$ & 18 \\
\hline SNS-032 & CDK & 0.88 & $\begin{array}{l}\text { Chronic lymphocytic } \\
\text { leukemia }\end{array}$ & 19 \\
\hline PIK-75 & PI3K & 0.86 & $\begin{array}{l}\text { Acute myeloid } \\
\text { leukemia }\end{array}$ & 20 \\
\hline GSK-461364 & PLK1, PLK3 & 0.76 & Solid tumors & 21 \\
\hline BI 2536 & PLK1 & 0.75 & Solid tumors & 22 \\
\hline Volasertib & PLK1 & 0.66 & Solid tumors & 23 \\
\hline BMS-3 & LIMK & 0.63 & Unknown & $\mathrm{N} / \mathrm{A}$ \\
\hline JNJ-10198409 & PDGFR & 0.6 & Glioblastoma & 24 \\
\hline Staurosporine & Pan kinases & 0.54 & Pan kinase inhibitor & 25 \\
\hline Dasatinib & bcr-Abl, Src & 0.52 & $\begin{array}{l}\text { Chronic myeloid } \\
\text { leukemia }\end{array}$ & 26 \\
\hline AZ-960 & JAKs & 0.51 & Adult T-cell leukemia & 27 \\
\hline
\end{tabular}

immunoblotting permit such determinations for averaged cell populations, but are labor intensive and slow. While imagebased techniques are amenable to a high-throughput report on individual cells and can report statistically significant changes, currently available image-based techniques require multiple steps, optimization, and are expensive, making them suboptimal for a drug screen aimed at providing biochemicalquality data (Fig. 2).

For a screen, the assay needs to be able to identify compounds as either active or inactive based on data from one sample (i.e., well), so any overlap in the distribution of response between active and inactive treatments results in false readouts. Moreover, the mechanism of cell death by any one compound may be very different than the positive control used in the screen. Ideally, the assay should not score stress responses as cytotoxic unless the stress is so acute that the cells will eventually die. Our results suggest that MVA-NAO fulfills all of these criteria as the dye is inexpensive, requires only a single addition step, no wash, and relatively little optimization. Due to the excitation sources available, we chose DRAQ5 as the DNA stain. Similar results would be obtained with a UV-based DNA stain (DAPI or Hoechst) on instruments capable of UV excitation, making this approach broadly applicable. Furthermore, the multivariate techniques needed to interpret the image data are commonly included in image processing software packages. 
The quality of a screening assay is typically measured through calculation of a $Z^{\prime}$ value based on high and low control treatments. ${ }^{14,15}$ In our hands, $24 \mathrm{~h}$ post-treatment, traditional measures of apoptosis such as cell count, nuclear shrinkage, and Annexin V staining all showed highly significant changes between our low controls (untreated) and high controls (TNF $\alpha$ ), but low $Z^{\prime}$ values (Table 2). Other measures gave better $Z^{\prime}$ values, such as measures of mitochondrial membrane potential and caspase activation, but the highest $Z^{\prime}$ values were obtained using MVA-NAO. As well as giving improved $Z^{\prime}$ values for $\mathrm{TNF} \alpha$ as a high control, MVA-NAO was able to identify toxicity induced by TG and BFA-known activators of cell death-at concentrations much lower than was detected by other image-based apoptosis assays (Fig. 2). In fact, only MVA-NAO detected activity in BFA at concentrations found to induce PARP cleavage in immunoblot.

In multivariate image analysis, a classifier is trained using features from cells known to be from the two classes to be separated. Selection of data for each class for training can be data from individual cells that a user has manually selected and identified ${ }^{16}$ or from a random subset taken from cells treated with or without known modulators of the response under study. Screening experiments are typically designed with in-plate controls, which inherently lend themselves to use as training data. In our case, we used the in-plate controls as training sets to remove the laborious manual classification step. Furthermore, to remove concerns about outlier cells affecting the reproducibility of the classifier when making random selections, we have taken a pragmatic approach using population averages from the high and low control wells as positive and negative controls, respectively, to train the classifier. This also simplifies and expedites the training of the classifier. While we used well-level averages to train the classifier, we used cell-level data, not well-level averages, for classification of unknown compounds. Using well-level averages would have resulted in a binary decision for the well and not have allowed sufficient granularity for ranking activity or derivation of dose-response curves.

Surprisingly, we found that a single activator of apoptosis (TNF $\alpha$ ) could be used to correctly, although with reduced sensitivity compared to the appropriate biochemical assay, classify activators of other cell death pathways. Comparison of the MVA-NAO data with the results of biochemical analyses for apoptosis, necrosis, endoplasmic reticulum stress, and autophagy suggests that when the classifier is trained on a positive control that kills cells exclusively by apoptosis, it is less sensitive at detecting cells dying by necrosis and does not incorrectly score endoplasmic reticulum stress or autophagy as cytotoxic unless the drug concentration is sufficiently high to kill cells. This is supported by the dose-response curve for tunicamycin, which does not plateau over the concentrations we have tested here.

Many drug trial failures and withdrawals can be attributed to unexpected organ toxicity. As such, predictive toxicity assays play a key role in drug development. Many idiosyncratic drug toxicities are correlated with mitochondrial function. Since NAO-related features will be correlated to mitochondrial function and structure, this assay should be useful for early identification of drug toxicity. ${ }^{17}$

\section{ACKNOWLEDGMENT}

This work was supported by CIHR grant FRN10490 to DWA.

\section{DISCLOSURE STATEMENT}

No competing financial interests exist.

\section{REFERENCES}

1. Galluzzi L, Bravo-San Pedro JM, Vitale I, et al.: Essential versus accessory aspects of cell death: recommendations of the NCCD 2015. Cell Death Differ 2015;22:58-73.

2. Kepp 0, Galluzzi L, Lipinski M, Yuan J, Kroemer G: Cell death assays for drug discovery. Nat Rev Drug Discov 2011;10:221-237.

3. Klionsky DJ, Abdalla FC, Abeliovich $H_{\text {, et }}$ al: Guidelines for the use and interpretation of assays for monitoring autophagy. Autophagy 2012;8:445-544.

4. Caie PD, Walls RE, Ingleston-Orme $A$, et al:: High-content phenotypic profiling of drug response signatures across distinct cancer cells. Mol Cancer Ther 2010;9:1913-1926.

5. Gustafsdottir SM, Ljosa V, Sokolnicki KL, et al.: Multiplex cytological profiling assay to measure diverse cellular states. PLoS One 2013;8:e80999.

6. Hamilton N, Pantelic R, Hanson K, Teasdale R: Fast automated cell phenotype image classification. BMC Bioinformatics 2007:8:110.

7. Murphy R, Velliste M, Porreca G: Robust numerical features for description and classification of subcellular location patterns in fluorescence microscope images. J Signal Process Syst. 2003;35:311-321.

8. Loo L-H, Lin H-J, Steininger RJ, Wang Y, Wu LF, Altschuler SJ: On an approach for extensibly profiling the molecular states of cellular subpopulations. Nat Methods 2009;6:759.

9. Loo L-H, Wu LF, Altschuler SJ: Image-based multivariate profiling of drug responses from single cells. Nat Methods 2007;4:445-453.

10. Haralick RM: Statistical and structural approaches to texture. Proc IEEE 1979;67:786-804.

11. Wu $Y$, Brodt $P$, Sun $H$, et al:: Insulin-like growth factor-I regulates the liver microenvironment in obese mice and promotes liver metastasis. Cancer Res 2010;70:57-67.

12. Manns J, Daubrawa $M$, Driessen $S$, et al:: Triggering of a novel intrinsic apoptosis pathway by the kinase inhibitor staurosporine: activation of caspase9 in the absence of Apaf-1. FASEB J 2011;25:3250-3261.

13. Ho C-Y, Wong C-H, Li H-Y: Perturbation of the chromosomal binding of RCC1, Mad2 and survivin causes spindle assembly defects and mitotic catastrophe. $J$ Cell Biochem 2008;105:835-846.

14. Iversen PW, Eastwood BJ, Sittampalam GS, Cox KL: A comparison of assay performance measures in screening assays: signal window, $Z^{\prime}$ factor, and assay variability ratio. J Biomol Screen 2006;11:247-252. 
15. Zhang J-H, Chung TDY, Oldenburg KR: A simple statistical parameter for use in evaluation and validation of high throughput screening assays. J Biomo/ Screen 1999;4:67-73.

16. Jones T, Kang I, Wheeler D, et al:: CellProfiler Analyst: data exploration and analysis software for complex image-based screens. BMC Bioinformatics 2008;9:482.

17. Xu JJ, Henstock PV, Dunn MC, Smith AR, Chabot JR, de Graaf D: Cellular imaging predictions of clinical drug-induced liver injury. Toxicol Sci 2008; 105:97-105.

18. Nagaria TS, Williams JL, Leduc C, Squire JA, Greer PA, Sangrar W: Flavopiridol synergizes with sorafenib to induce cytotoxicity and potentiate antitumorigenic activity in EGFR/HER-2 and mutant RAS/RAF breast cancer model systems. Neoplasia 2013;15:939-951.

19. Misra RN, Xiao HY, Kim KS, et al:: N-(cycloalkylamino)acyl-2-aminothiazole inhibitors of cyclin-dependent kinase 2. N-[5-[[[5-(1,1-dimethylethyl)-2oxazolyl]methyl]thio]-2-thiazolyl]-4- piperidinecarboxamide (BMS-387032), a highly efficacious and selective antitumor agent. J Med Chem 2004;47:17191728.

20. Talekar M, Ganta S, Amiji M, et al.: Development of PIK-75 nanosuspension formulation with enhanced delivery efficiency and cytotoxicity for targeted anti-cancer therapy. Int J Pharm 2013;450:278-289.

21. Wu CP, Hsiao SH, Luo SY, et al.: Overexpression of human ABCB1 in cancer cells leads to reduced activity of GSK461364, a specific inhibitor of polo-like kinase 1. Mol Pharm 2014;11:3727-3736.

22. Ansbro MR, Shukla S, Ambudkar SV, Yuspa SH, Li L: Screening compounds with a novel high-throughput ABCB1-mediated efflux assay identifies drugs with known therapeutic targets at risk for multidrug resistance interference. PLoS One 2013;8:e60334.

23. Rudolph D, Steegmaier M, Hoffmann M, et al:: BI 6727, a Polo-like kinase inhibitor with improved pharmacokinetic profile and broad antitumor activity. Clin Cancer Res 2009;15:3094-3102.

24. Ho CY, Ludovici DW, Maharoof US, et al:: (6,7-Dimethoxy-2,4-dihydroindeno[1,2c]pyrazol-3-yl)phenylamines: platelet-derived growth factor receptor tyrosine kinase inhibitors with broad antiproliferative activity against tumor cells. J Med Chem 2005:48:8163-8173

25. Cyr L, Langler R, Lavigne C: Cell cycle arrest and apoptosis responses of human breast epithelial cells to the synthetic organosulfur compound $p$ methoxyphenyl p-toluenesulfonate. Anticancer Res 2008;28:2753-2763.
26. Chen $T$, Wang $C$, Liu 0 , et al:: Dasatinib reverses the multidrug resistance of breast cancer MCF-7 cells to doxorubicin by downregulating P-gp expression via inhibiting the activation of ERK signaling pathway. Cancer Biol Ther 2015;16:106-114.

27. Yang J, Ikezoe T, Nishioka C, Furihata M, Yokoyama A: AZ960, a novel Jak2 inhibitor, induces growth arrest and apoptosis in adult T-cell leukemia cells. Mol Cancer Ther 2010;9:3386-3395.

Address correspondence to:

David W. Andrews, PhD

Department of Biological Sciences

Sunnybrook Research Institute 2075 Bayview Avenue

Toronto, ON M4N 3M5

Canada

E-mail: david.andrews@sri.utoronto.ca

\section{Abbreviations Used}

$$
\begin{aligned}
\alpha-\mathrm{MEM} & =\alpha \text {-minimal essential medium } \\
\mathrm{BFA} & =\text { brefeldin } \mathrm{A} \\
\mathrm{DMSO} & =\text { dimethylsulfoxide } \\
\mathrm{DTT} & =\text { dithiothreitol } \\
\mathrm{Grp} & =\text { glucose-regulated protein } \\
\mathrm{LC} 3 & =\text { light chain } 3 \\
\mathrm{mCer} & =\text { monomeric Cerulean } \\
\mathrm{NAO} & =\text { nonyl acridine orange } \\
\mathrm{PARP} & =\text { poly-ADP ribose polymerase } \\
\mathrm{PI} & =\text { propidium iodide } \\
\mathrm{SVM} & =\text { support vector machine } \\
\mathrm{TG} & =\text { thapsigargin } \\
\mathrm{TMRE} & =\text { tetramethylrhodamine }, \text { ethyl ester } \\
\mathrm{TNF} \alpha & =\text { tumor necrosis factor-alpha }
\end{aligned}
$$

